\title{
Immersive Virtual Reality for Clinical Pain: Considerations for Effective Therapy
}

\author{
Tony Donegan ${ }^{1 *}$, Brenda E. Ryan ${ }^{1}$, Justyna Swidrak ${ }^{1,2,3}$ and Maria V. Sanchez-Vives ${ }^{1,2,4,5}$ \\ ${ }^{1}$ Institut d'Investigacions Biomèdiques August Pi i Sunyer (IDIBAPS), Barcelona, Spain, ${ }^{2}$ Event-Lab, Barcelona, Spain, \\ ${ }^{3}$ Institute of Psychology, Polish Academy of Sciences, Warsaw, Poland, ${ }^{4}$ Institució Catalana de Recerca i Estudis Avançats \\ (ICREA), Barcelona, Spain, ${ }^{5}$ Department of Cognition, Development and Educational Psychology, University of Barcelona, \\ Barcelona, Spain
}

Immersive virtual reality is transforming medical and psychological research and treatment, including the treatment of clinical pain. In this short perspective paper, we present some of the methodological difficulties that are rarely discussed in the literature of pain research when using virtual reality. These often-unmentioned problems can confound research investigations or interfere with the therapeutic efficacy in clinical trials. We propose practical solutions based on our research experience. We first outline the mechanisms of, and challenges to, the sensations of embodiment and presence, which are critical to creating effective virtual reality illusions, before discussing the particular considerations that need to be contemplated when working with patients with clinical pain. Finally, we discuss some upcoming technological advances that may influence significantly this rapidly expanding field in the near future.

Keywords: virtual reality, pain, embodiment, presence, body ownership, HMD, immersive, virtual rehabilitation

\section{INTRODUCTION}

Immersive virtual reality (VR) is a computer-generated 3D environment that fully surrounds the user and that is displayed in a head-tracked mounted display (HMD) or, less commonly, in a cave automatic virtual environment (CAVE). The virtual environment typically provides a $360^{\circ}$ virtual scene which can transport the user to an artificial world and even give them a virtual body with which to explore it. This artificial world and body can be freely controlled and manipulated by the user, allowing for the experience of environments and situations that are simulations of reality, or of environments and situations that would be impossible, too dangerous or even ethically problematic in reality. VR has applications in real life beyond gaming, including education and psychological and medical research and treatment (for a review see Slater and Sanchez-Vives, 2016). Until relatively recently VR systems cost tens of thousands of euros, and their use was largely restricted to research centers and specialist laboratories, out of reach to the general public. Nowadays, costs are dramatically lower, with standalone headset systems which are available for less than $€ 500$. This opens up the possibility for the ubiquitous use of VR not only in entertainment, but also in more diverse areas including the hospital environments and in the home (Slater, 2014). Thus, in addition to gaming, educational and training applications, there is considerable potential for the use of VR as a therapeutic tool in the fields of physical rehabilitation, mental health, or pain management or treatment. 
While the use of immersive VR for the management of acute pain conditions has been relatively well-established (e.g., Schmitt et al., 2011; Soltani et al., 2018), the evidence to support the use of VR in chronic pain is less conclusive. While much preliminary and proof-of-concept research shows promise (e.g., Harvie et al., 2015; Matamala-Gomez et al., 2018; Stanton et al., 2018), rigorous randomized controlled trials with larger samples are required for a consistent demonstration that chronic pain treatment in virtual environments is superior to conventional treatments (Crosbie et al., 2007; Boesch et al., 2015), and many such trials are already in development.

Generally, therapeutic VR is considered low risk and no adverse events have been reported after therapeutic VR (in a meta-analyses of 11 studies) (Corbetta et al., 2015). However, patients with acute or chronic clinical pain that use therapeutic VR are (by definition) in suboptimal health: as well as being in pain, they often have reduced mobility, they may tire easily, and their ability to concentrate for more than short periods can be affected, not just as a result of their condition but also potentially the side-effects of any pain-relieving medication, or from other co-morbidities. Such patients require particular considerations when designing, testing and implementing treatment using immersive VR. Our own experiences with using therapeutic VR has involved, collectively, hundreds of patients with clinical pain, and this has allowed us to informally garner a series of patient perspectives that help to outline the practicalities and challenges associated with its use. In this short paper, we present these challenges and offer practical solutions. Our intention is to give some guidance to researchers and therapists when designing experiments and treatments in order to induce an effective immersive experience as well as maximize patient comfort and safety.

\section{CHALLENGES TO EFFECTIVE THERAPEUTIC IMMERSIVE VR}

For VR to be an efficient therapeutic tool, several phenomena need to take place. Firstly, patients need to feel that they are located in the virtual scene (presence illusion) and that the events experienced in the virtual environment are plausible (plausibility illusion) (Slater, 2009). Secondly, and depending on the application (e.g., Perez-Marcos et al., 2012), it may be relevant that the virtual body that the patient sees is their own body (embodiment illusion; Slater et al., 2009; Sanchez-Vives et al., 2010). Here we describe some of the barriers that may impede these illusions and suggest some solutions to them. These barriers are also summarized in Table 1.

\section{Challenges to the Sense of Embodiment}

\section{"It felt like someone else's arm, not mine." "It felt like I had a third arm"}

In VR terminology, "embodiment" refers to the illusory ownership of a virtual body. Our sense of embodiment is highly malleable experimentally, and we can readily embody bodies that are radically different from our own real-life bodies using VR (see Kilteni et al., 2012; Slater and Sanchez-Vives, 2016). Since chronic pain is associated with cortical changes that manifest in altered body image (Lotze and Moseley, 2007), the manipulation of body image using embodiment in VR is a viable therapeutic avenue that is currently under further exploration (for a review see Matamala-Gomez et al., 2019).

This sense of embodiment can be increased through correlated multisensory input, which may be mediated through one or more of the following:

1. Co-location of the virtual and real limbs, so that visual and proprioceptive feedback regarding body position match (Nierula et al., 2017);

2. Visuomotor feedback, in which real-life body movements are matched by those of the virtual avatar (mediated through tracking via the HMD or external sensors) (Sanchez-Vives et al., 2010); or

3. Visuotactile feedback, in which contact of a virtual object with the virtual body is emphasized through concurrent tactile sensation at the matching location in real life (Slater et al., 2008).

From a predictive processing perspective (Clark, 2016), when inducing a sense of embodiment the precision weighting accorded to vision is higher than for other sensations such as proprioception (Bourdin et al., 2019), touch (Slater et al., 2008) or temperature (Llobera et al., 2013). However, it seems that when there is at least one other form of congruent sensory input, the embodiment illusion is significantly more powerful than with vision alone (Botvinick and Cohen, 1998; Azañón et al., 2016; Marasco, 2018), with visuomotor congruence seemingly more powerful than visuotactile (Kokkinara and Slater, 2014). Any disruption to the congruency of these sensations can reduce or break this sense of embodiment. Indeed, our patients have occasionally reported phenomena associated with incomplete sensations of embodiment, such as owning a supernumerary limb (a third arm), or that their virtual arms belonged to someone standing behind them.

\section{Agency}

In patients with an inability or unwillingness to move their painful body or body part (e.g., through immobilization, in cases of fracture or stroke; or through fear-avoidance behaviors in cases of painful conditions), it can be more difficult to induce an illusion of ownership or sense of agency over the virtual limb than in healthy participants who move normally (Sanchez-Vives et al., 2010). There are a number of potential solutions to this, such as induction of ownership by means of visuotactile congruence (Slater et al., 2008). Another option is to use the movement of the contralateral real limb, which could be tracked with the movement transferred to the ipsilateral virtual limb (in a manner similar to traditional mirror therapy).

With visuomotor congruence, discrepancies of more than $150 \mathrm{~ms}$ between virtual and real movement are thought to reduce the sense of agency (Kilteni et al., 2012). However, there is some tolerance regarding the actual location of the moved arm (Bourdin et al., 2019). As technologies and processing speeds continue to improve, this latency is less and less likely to be 
TABLE 1 | A summary of selected challenges and solutions in VR studies related to embodiment and presence.

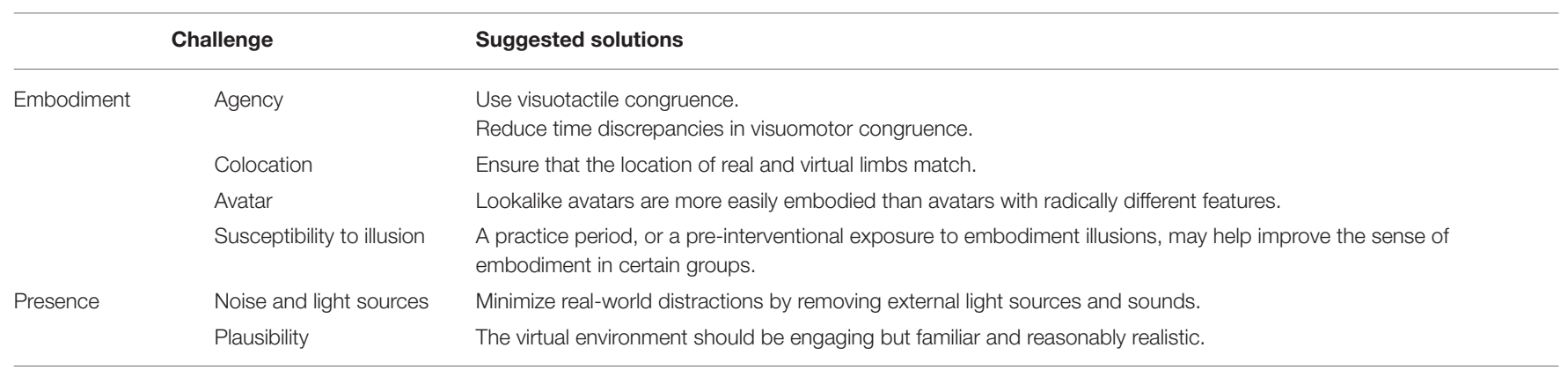

an issue; however, researchers should always ensure that the system runs smoothly and the hardware exceeds the minimal specifications required to handle VR, given that experimental participants or patients rarely report this.

\section{Colocation}

The illusion of ownership of a rubber arm (Botvinick and Cohen, 1998) or virtual arm can be achieved in the absence of colocation. However, colocation of the real body and the virtual body has advantages. Nierula et al. (2017) showed that the analgesic effects of viewing a painful virtual body part are reduced if there is a discrepancy of more than $30 \mathrm{~cm}$ between the real and virtual limbs. Researchers should therefore ensure the location of real and virtual limbs match. In systems with body tracking this may happen automatically, unless programmed otherwise. Accurate co-location may be problematic in amputee patients with phantom limb pain, as they often experience their phantom limb to be located in awkward positions, and can also present with telescopic shortening of the limb, phenomena that cannot easily be replicated in real time in VR.

\section{Groups With Reduced Susceptibility to Embodiment Illusions}

Our experience is that participants with a high level of proprioceptive acuity, such as gymnasts, yoga practitioners, and elite-level athletes, find it more difficult to embody a virtual body. We speculate that their intense training results in their brain giving a comparatively greater predictive weighting to proprioceptive input, so that they rely less on the visual input given by the HMD when attempting to produce a prediction regarding their body's spatial location (Bourdin et al., 2019).

It is also our experience that embodiment levels, as measured by questionnaire, seem to be lower in more elderly patients. This is coherent with results in the literature with the rubber hand embodiment illusion (Ferracci and Brancucci, 2019). A possible explanation is that elderly people may rely less on bottom-up sensory input, and more on top-down (i.e., bodily representational) influences-essentially, their body image may be more rigid. That said, in our experience the ability to embody a virtual body or body part seems to improve with practice. A practice period, or a pre-interventional exposure to embodiment illusions, may therefore help improve the sense of embodiment in such patients.

\section{Challenges to the Sense of Presence}

The illusion of being transported to the place depicted by the virtual environment, known as the "sense of presence" (Slater, 2009), has a powerful distraction effect, and appears to be a key component of the modulation of acute pain (Hoffman et al., 2000). The sense of presence results from integrating two factors: place illusion and plausibility of the content. We have found that the illusion of being in the place depicted in VR can be easily disrupted.

\section{External Noise and Light Sources}

In order to maintain a strong presence illusion in the virtual environment, it would seem prudent to minimize real-world distractions by removing external light sources and sounds, including communication between researcher and patient if possible.

\section{Correlated Physical and Virtual World}

Encountering or bumping into objects that are non-existent in the virtual environment can often break the sense of presence. Patients will not often mention that they encounter this problem and it may go unnoticed by the researcher. Researchers should always personally try the environments and keep the "playing area" of the experience free of cables or objects that are not represented visually in the virtual environment.

\section{Plausibility of Content}

The observed virtual environment should be at once engaging yet familiar and reasonably realistic in order to ensure an effective plausibility illusion and sense of presence, which is thought to encourage normal behavior (Slater, 2009). Most importantly, if the environment is meant to be depicting something that could occur in reality, then it should be consistent with expectations of the patients.

\section{General Considerations for Patients in Pain}

Some general considerations when working with people in pain are outlined in Table 2, and discussed below.

\section{Exposure Time and Fatigue}

"I cannot relax anymore like I did in the beginning"
"It's exhausting, concentrating for that long" 
TABLE 2 | A summary of selected challenges and solutions in VR studies with special considerations for patients with pain.

\begin{tabular}{|c|c|}
\hline Challenge & Suggested solutions \\
\hline $\begin{array}{l}\text { Exposure time and } \\
\text { fatigue }\end{array}$ & $\begin{array}{l}\text { Set the tasks at an appropriate level for the patient. } \\
\text { Ensure a graded progression of difficulty. } \\
\text { Exposure time should be kept to a maximum 10-15 min. } \\
\text { Encourage patients beforehand to report adverse symptoms immediately and discontinue the treatment. } \\
\text { Repeated exposure may evoke a habituation effect and patients may cope with longer sessions with training. }\end{array}$ \\
\hline Nausea, dizziness & $\begin{array}{l}\text { Keep the visual and vestibular mismatches to a minimum. } \\
\text { Use of airflow; slowing down the movements; narrowing the field of view; ensuring the interpupillary distance (IPD) is correct. }\end{array}$ \\
\hline Hygiene & $\begin{array}{l}\text { Use single-use disposable VR masks. } \\
\text { Wipe down and disinfect hard surfaces between patients. } \\
\text { Replace sponge elements of the HMDs with a vinyl cover. } \\
\text { Regular handwashing }\end{array}$ \\
\hline $\begin{array}{l}\text { Technophobia, lack of } \\
\text { trust }\end{array}$ & $\begin{array}{l}\text { Discuss beforehand what is expected from the participant. } \\
\text { Spend some time answering the participant's questions and doubts. } \\
\text { Give the participants the possibility to explore the equipment and test a virtual environment before the experiment starts. } \\
\text { Show a "virtual therapist" that provides instruction and encouragement to the participants. } \\
\text { Set demands of the task based on the participant's expertise in virtual environments and with video games. }\end{array}$ \\
\hline Lack of movement & $\begin{array}{l}\text { Position patients into comfortable positions prior to starting any treatment. } \\
\text { Encourage the participants to fidget, shuffle and change position regularly if they wish to. }\end{array}$ \\
\hline Excessive movement & $\begin{array}{l}\text { Avoid end of range motion (particularly of the neck). } \\
\text { Consider carefully the patient's condition, and tailor the experience according to the amount and type of movement } \\
\text { available to the patient. }\end{array}$ \\
\hline Headset weight & $\begin{array}{l}\text { Encourage patient feedback regarding neck pain and consider shorter sessions in these patients. } \\
\text { Support the head and neck by providing neck pillows or raised back supports if required. }\end{array}$ \\
\hline Patients with allodynia & $\begin{array}{l}\text { Avoid tactile feedback over the area of allodynia. } \\
\text { Explore alternative multisensory congruence. } \\
\text { Interoceptive information such as heartbeat, together with vision, can be used. }\end{array}$ \\
\hline Questionnaires & Choose shorter questions with clearer language, minimize the use of abstract terminology. \\
\hline
\end{tabular}

Spending time VR has the potential to induce symptoms not dissimilar to motion sickness, such as nausea, dizziness, headache, and fatigue (LaViola, 2000). However, in our experience with at least 400 participants and exposures of $15 \mathrm{~min}$, this has hardly ever been the case (provided that we do not use high-speed environments such as rollercoaster simulations). Many patients, particularly those with fibromyalgia, stroke patients, post-operative patients, or those on strong pain medication, often experience symptoms of mental fatigue when having to concentrate for long periods. VR can require significant mental resources (depending on the task demand and the level of interaction required), which can often overwhelm patients unaccustomed to it. Careful and considered treatment planning, which includes setting the tasks at an appropriate level for the patient, as well as ensuring a graded progression of difficulty, can help avoid these problems. For patients in pain, who will probably fatigue easily, we recommend that the time of each exposure should be kept to $10-15$ min per session at the most. Patients should be encouraged beforehand to report any such symptoms immediately and the treatment discontinued. On the other hand, repeated exposure may evoke a habituation effect and patients may cope with longer sessions with training (Hill and Howarth, 2000; Howarth and Hodder, 2008).

\section{Nausea and Dizziness}

"The ball moves too fast and makes me feel sick"
Any mismatch between sensory input from the visual and proprioceptive systems has the potential to cause a form of motion sickness. In VR, this can occur when the visual perception of self-motion is not matched by reality (LaViola, 2000). Some suggested solutions include the use of airflow (D'Amour et al., 2017), slowing down the movements (Kemeny et al., 2017), narrowing the field of view (Fernandes and Feiner, 2016), and ensuring the interpupillary distance (IPD) is correct (Fulvio et al., 2019). As discussed, it is also thought that as patients adapt over time to being in the virtual environment, so the severity of cybersickness may be reduced (Hill and Howarth, 2000; Howarth and Hodder, 2008). Again, keeping the visual and vestibular mismatches to a minimum, especially in the early stages of treatment, would seem prudent.

\section{Hygiene}

Most commercial HMDs utilize felt or sponge lining that, while comfortable, makes sterilization of the headset difficult. This may be problematic in a hospital setting where oftenstrict sterilization protocols need to be met, in particular if used by oncological or immunosuppressed patients (this is particularly pertinent in the current COVID-19 crisis). If the same HMD is to be used by many people, potential solutions include single-use disposable VR masks, the use of ultraviolet radiation boxes, and following standard sterilization protocols including wiping down and disinfecting hard surfaces between patients and frequent hand-washing. Additionally, it is possible 
to replace the original sponge elements of the HMDs with a vinyl one.

\section{Technophobic Patients}

$$
\text { "I'm too old for this" }
$$

For some participants, the experimental situation can be intimidating, especially if they do not have any previous experience with VR or video games. Some patients struggle with exploring the virtual environment spontaneously and need verbal encouragement in order to do so. This is particularly noticeable with elderly participants. A thorough discussion beforehand of what is expected of the participant during the experiment, including giving them the possibility to explore the equipment and test a virtual environment before the experiment starts is helpful. Another option is to have a virtual therapist (preferably embodied in a third-person avatar) that provides instruction and encouragement, and in our experience patients often respond well to this while in the virtual environment.

Many patients are worried that they will fail, break the equipment, or be seen by those observing as not being clever or quick enough, or that they are too old for it. It is worthwhile spending extra time explaining the equipment and answering any questions and doubts. Patients respond best to healthcare treatments when an effective therapeutic relationship has been established, and there is no reason VR treatment should be any different. A recent study examined the experience of elderly participants involved in a participatory design process; that is, including participants in all stages of virtual environment design (Kopeć et al., 2019), with the results suggesting that such a process may be useful to combat these fears and significantly enhance the patient experience. In our experience, once a potential rejection is overcome, older participants commonly enjoy using VR and they are enthusiastic subjectsas most naïve VR patients are-both for experiments and for therapeutic interventions.

\section{Avatar Characteristics}

$$
\text { "It's me!" }
$$

In VR, participants can readily embody avatars that are quite different from their real-life body and the characteristics of this virtual body can have marked psychological effects (see, e.g., Maister et al., 2015; Banakou et al., 2016). However, unless manipulation of the virtual body is a particular focus of the treatment, it makes sense to try to match the physical characteristics of the patient and the avatar. Mapping of patient's real-life facial characteristics is becoming more straightforward, and patients are often pleased to see an accurate "lookalike" avatar when they look into a virtual mirror, and as a result they embody the lookalike more easily than an unfamiliar avatar.

\section{Appropriate Task Demand}

\author{
"What do I have to do now?" \\ "I had to close my eyes - it was all a bit overwhelming!"
}

Knowing the participant's expertise beforehand can be useful to select demands of the task they will be facing in the virtual environment. As mentioned previously, it is important that virtual therapy (as with any therapy) starts easy and builds gradually, in terms of the difficulty of task performed, the level of movement involved, and the time spent in the virtual environment. Patients will have different expectations regarding what will happen in VR-more experienced participants faced with low-demanding-behavioral environments may feel bored or confused; less experienced participants may be overwhelmed. The ability to tailor a starting point based on the patient's perceived expertise in virtual environments and with video gaming may therefore be helpful.

\section{Lack of Movement}

"It is starting to hurt again because I can't move my hands and I'm tightening them up".

Patients are often in a static body position throughout their VR experience. Even with head/neck and upper limb movements, patients do not actually move from a seated or recumbent position. Indeed, this is often done for reasons of safety, since immersion in the virtual environment can be quite disorientating, particularly with first use. However, this prolonged lack of movement can exacerbate certain pain conditions. Comfort is king, and patients should be positioned into comfortable positions prior to starting any treatment, with encouragement to fidget, shuffle and change position regularly if they wish to. We have found that generally patients do not tolerate a long-sitting position particularly well, compared with a standard seating position. This may be due to the excessive mechanical tension of the nervous system involved in the longsitting position.

\section{Excessive Movement}

"I think I hurt my neck looking around!"

Conversely, extreme end of range motion (particularly of the neck) should generally be avoided unless absolutely necessary. However, such movements, if required, can be visualized in VR without being performed in real life, often without the participant noticing (Harvie et al., 2015; Bourdin et al., 2019). Careful consideration of the patient's condition, and a prediction of the amount and type of movement involved, is required in order to ensure that the patient's symptoms are not exacerbated by the treatment, and a basic functional assessment examining global range of movement and movement capacity can be carried out by the researcher to assess potential exacerbating factors prior to treatment (ideally by a physiotherapist).

\section{Headset Weight}

Some HMDs weigh little and are easy to adjust whilst others are comparatively heavy and bulky. This may cause neck pain and discomfort, particularly with prolonged use, and particularly in patients with pre-existing neck pain or fatigue issues. Patient feedback regarding neck pain should be encouraged and shorter 
sessions considered in these patients. Supporting the head and neck by providing neck pillows, or raised back supports is also worth considering; although these can be restrictive of neck movements and may cause a visuotactile mismatch that can break the embodiment illusion.

\section{Patients With Allodynia}

"I can't handle it [vibrotactile stimulation] any more. It feels like needles."

Tactile feedback is often given using vibration, in order to provide congruent multisensory input and induce an embodiment illusion (see Challenges to the Sense of Embodiment). However, in some pain conditions (e.g., complex regional pain syndrome), normal tactile input can be processed as painful, a phenomenon of central sensitization known as allodynia. Alternative multisensory congruence is therefore preferable, but difficult to achieve. Movement of the limb is also often extremely painful for these patients, which effectively rules out using visuomotor congruence; and co-location of the real and virtual bodies (visuoproprioceptive feedback) has a weaker effect on inducing body ownership than when providing congruent visuotactile and/or visuomotor sensory input. However, other possibilities could be explored, such as tactile feedback on a non-allodynic area or other congruent sensory modalities. Another potential alternative is to use interoceptive information such as heartbeat, together with vision, to provide this input (Solcà et al., 2018).

\section{Subjective Outcome Measures (Questionnaires)}

\author{
"I don't know if I fully understand this question" \\ "What does it mean that the body belonged to someone else?"
}

Some questions, especially related to abstract concepts like embodiment or presence, are often confusing for patients unfamiliar with such terms. We have found that patients unfamiliar with a Likert or sliding-scale questions often respond as if the scale were dichotomous and select only the extreme options. Moreover, long and complex questions seem to discourage and distract patients, and patients sometimes give answers that seem to be inconsistent with their informally reported experience.

Our recommendation is to choose shorter questions with clearer language, minimizing the use of abstract terminology. However, reducing the number of possible answers to simplify matters for the patient, then runs the risk of reducing the sensitivity of the measure. While there is currently no widely used standardized questionnaire for measuring embodiment levels, Gonzalez-Franco and Peck (2018) have proposed a comprehensive 25-question outcome measure that covers six different domains (body ownership, agency and motor control, tactile sensations, bodily location, external appearance, and response to external stimuli). However, validation of such measures is problematic since there is no gold standard.

\section{FUTURE DEVELOPMENTS IN THERAPEUTIC VR}

The market of immersive VR devices is growing rapidlycertain aspects of VR technology that were unthinkable only a few years ago have become commonplace today (Coburn et al., 2017; Riva et al., 2018; Sherman and Craig, 2018). Future technological developments have the potential to further improve therapeutic VR. For patients, perhaps the most significant is fullbody tracking in a standalone HMD, which is expected in the next generation of HMDs. A system with full-body tracking that does not require any special hardware would make the setup much faster, programming easier, and would be significantly more patient-friendly, thanks to a reduction in the amount of equipment to set up and calibrate. For technophobic patients and patients with significantly reduced mobility, this ease of setup is often critical. The increased portability of the system means that patients have much more flexibility with where and when they can receive treatment.

For patients with severely impaired motor control, for example due to a stroke, spinal cord injury, or peripheral nerve injuries, or for amputees (conditions which can be also associated with pain), a potentially promising solution is the use of braincomputer interfaces (BCI) in combination with VR (Nierula et al., 2019; Leeb and Pérez-Marcos, 2020). Leeb et al. (2012) demonstrated for the first time that it is possible to navigate in VR by asynchronous, self-pace-controlled VR. Previously, PerezMarcos et al. (2009) demonstrated that the control of virtual hand movement by motor imagery was effective at inducing ownership of the virtual hand, when compared to the same set-up with a lack of control (or incongruent condition). Such control implies an activity in the motor cortex that results in a visual feedback of the desired action, therefore there are correct sensorimotor congruence that induce ownership of the virtual body part and agency over it. There are other BCI protocols that can be used to control the movement of the virtual body (or eventually of other features of the virtual environment). A commonly used one is visual-evoked potentials. Our studies, however, have found that the sense of agency and responsibility over movements of a virtual body are larger when triggered from the sensorimotor cortex than from visual-evoked potentials in healthy participants (Nierula et al., 2019). VR-BCI confers various advantages over BCI alone (Leeb and Pérez-Marcos, 2020). It has the potential to be more ecologically valid, more versatile, and can provide more motivational feedback for BCI users. Additionally it is safe, cost-effective, and flexible (e.g., testing a wheelchair without any risk) (Lotte et al., 2012). The use of motor imagery in VR-BCI is a good example of how these two technologies can potentially be combined. Although a large part of the research combining these techniques has been in healthy participants (e.g., Ortner et al., 2012; Lupu et al., 2018; Nierula and Sanchez-Vives, 2019; Nierula et al., 2019), or is still at proof-of-concept stage (Aamer et al., 2019), early clinical studies have shown promise for neurorehabilitation (Remsik et al., 2018; Cohen et al., 2019; Vourvopoulos et al., 2019). However, the spatiotemporal imprecision is currently a limiting factor. 
Perhaps the greatest limitation of current VR technology is the lack of a realistic touch sensation, known as haptics. Interpersonal mediated touch is known for its many therapeutic effects (e.g., Cabibihan et al., 2012; Serrano et al., 2016; Spiss et al., 2018), and it is little wonder that many research laboratories, both in academia and in industry, are testing potential solutions. Currently, the most commonly applied solution is the use of vibrators located on velcro strips, which lack the richness and variety of physical contact (warmth, softness, pressure, etc.). Touch stimuli can be processed and interpreted in numerous different ways depending on the context of the experience and the meaning attributed to it, with visual stimulation being a strong contextual modulator of touch sensation; however, a completely realistic feeling of touch is much more difficult to achieve. Some full-body suits (e.g., Teslasuit) use electrostimulation channels which are purported to enrich the stimulation of vibrators.

Perhaps the more pertinent questions are scientific and not technical. There is some research to suggest that the most effective strategy when using VR for pain relief may need to be tailored to the pathology (Matamala-Gomez et al., 2018), and may even be individually specific (Stanton et al., 2018). The optimal dosage to maximize pain relief, and whether analgesic effects are sustained over time are also unclear but should be elucidated over the coming few years. For chronic pain in particular, designing an individually specific intervention that addresses the unique and complex multidimensional aspects of the patient's lived experience of pain may be one of the biggest challenges both for VR developers, just as it is for therapists, but will ultimately be essential if therapeutic VR is to have clinically meaningful effects. At the same time, as the technology becomes cheaper and more accessible, personalization may also be seen as one of VR therapy's strengths, with aspects such as real-time feedback and tailored environments and avatars being particularly useful.

\section{CONCLUSION}

Immersive VR technology has opened a brand-new chapter in rehabilitation and pain treatment and has garnered the interest among a rapidly and constantly growing circle of researchers, doctors, and physiotherapists. We have introduced some of the

\section{REFERENCES}

Aamer, A., Esawy, A., Swelam, O., Nabil, T., Anwar, A., and Eldeib, A. (2019). "BCI integrated with VR for rehabilitation," in Proceedings of the International Conference on Microelectronics, ICM (Cairo). doi: 10.1109/ICM48031.2019.90 21752

Azañón, E., Tamè, L., Maravita, A., Linkenauger, S. A., Ferrè, E. R., Tajadura-Jiménez, A., et al. (2016). Multimodal contributions to body representation. Multisens. Res. 29, 635-661. doi: 10.1163/22134808-000 02531

Banakou, D., Hanumanthu, P. D., and Slater, M. (2016). Virtual embodiment of white people in a black virtual body leads to a sustained reduction in their implicit racial bias. Front. Hum. Neurosci. 10:601. doi: 10.3389/fnhum.2016. 00601 potential problems or risks that may hamper research efforts and proposed some solutions which are often omitted in the literature but can be helpful for those who would like to begin or have just begun working with immersive VR. The challenges outlined are not intended to be (nor could ever be) an exhaustive list, and our experiences merely serve to highlight the fact that each patient is different, with a unique biopsychosocial profile, and effective treatments within and without VR are those that recognize and accommodate the complexity and individuality of the patient and their pain experience.

\section{ETHICS STATEMENT}

The studies involving human participants were reviewed and approved by the ethics committees of Hospital Clinic de Barcelona. Written informed consent to participate in this study was provided by the patients. Written informed consent was obtained from the individual for the publication of any potentially identifiable images or data included in this article.

\section{AUTHOR CONTRIBUTIONS}

All authors listed have made a substantial, direct and intellectual contribution to the work, and approved it for publication.

\section{FUNDING}

This work was supported by NEUROVIRTUAL 2017-SGR01296, and by CECH, funded by GENCAT_TechEmergent18 by the Commission for Universities and Research of the Department of Innovation, Universities, and Enterprise of the Generalitat de Catalunya -AGAUR- (IU16-011508) and cofinanced by the European Union Regional Development Fund within the framework of the ERDF/FEDER Operational Program of Catalonia 2014-2020 to MVS-V.

\section{ACKNOWLEDGMENTS}

The authors would like to thank Mel Slater for his advice and helpful comments. 
Clark, A. (2016). Surfing Uncertainty: Prediction, Action, and the Embodied Mind. Oxford, MS: OUP. doi: 10.1093/acprof:oso/9780190217013.001.0001

Coburn, J. Q., Freeman, I., and Salmon, J. L. (2017). A review of the capabilities of current low-cost virtual reality technology and its potential to enhance the design process. J. Comput. Inf. Sci. Eng. 17:031013. doi: 10.1115/1.40 36921

Cohen, O., Doron, D., Koppel, M., Malach, R., and Friedman, D. (2019). "High performance BCI in controlling an avatar using the missing hand representation in long term amputees," in Brain-Computer Interface Research, eds C. Guger, N. Mrachacz-Kersting, and B. Z. Allison (Cham: Springer), 93-101. doi: 10.1007/978-3-030-05668-1_9

Corbetta, D., Imeri, F., and Gatti, R. (2015). Rehabilitation that incorporates virtual reality is more effective than standard rehabilitation for improving walking speed, balance and mobility after stroke: a systematic review. J. Physiother. 61, 117-124. doi: 10.1016/j.jphys.2015.05.017

Crosbie, J. H., Lennon, S., Basford, J. R., and McDonough, S. M. (2007). Virtual reality in stroke rehabilitation: still more virtual than real. Disabil. Rehabil. 29, 1139-1146. doi: 10.1080/09638280600960909

D'Amour, S., Bos, J. E., and Keshavarz, B. (2017). The efficacy of airflow and seat vibration on reducing visually induced motion sickness. Exp. Brain Res. 235, 2811-2820. doi: 10.1007/s00221-017-5009-1

Fernandes, A. S., and Feiner, S. K. (2016). "Combating VR sickness through subtle dynamic field-of-view modification," in IEEE Symposium on 3D User Interfaces (Greenville, SC: IEEE). doi: 10.1109/3DUI.2016.7460053

Ferracci, S., and Brancucci, A. (2019). The influence of age on the rubber hand illusion. Conscious. Cogn. 73:102756. doi: 10.1016/j.concog.2019.05.004

Fulvio, J. M., Ji, M., and Rokers, B. (2019). Variability in sensory sensitivity predicts motion sickness in virtual reality. bioRxiv [Preprint] 488817. doi: $10.1101 / 488817$

Gonzalez-Franco, M., and Peck, T. C. (2018). Avatar embodiment. Towards a standardized questionnaire. Front. Robot. AI 5:74. doi: $10.3389 /$ frobt.2018.00074

Harvie, D. S., Broecker, M., Smith, R. T., Meulders, A., Madden, V. J., and Moseley, G. L. (2015). Bogus Visual feedback alters onset of movement-evoked pain in people with neck pain. Psychol. Sci. 26, 385-392. doi: $10.1177 / 0956797614563339$

Hill, K. J., and Howarth, P. A. (2000). Habituation to the side effects of immersion in a virtual environment. Displays 21, 25-30. doi: 10.1016/S0141-9382(00)00029-9

Hoffman, H. G., Patterson, D. R., and Carrougher, G. J. (2000). Use of virtual reality for adjunctive treatment of adult burn pain during physical therapy: a controlled study. Clin. J. Pain 16, 244-250. doi: 10.1097/00002508-200009000-00010

Howarth, P. A., and Hodder, S. G. (2008). Characteristics of habituation to motion in a virtual environment. Displays 29, 117-123. doi: 10.1016/j.displa.2007.09.009

Kemeny, A., George, P., Merienne, F., Colombet, F., and Mérienne, F. (2017). "New VR navigation techniques to reduce cybersickness," in The Engineering Reality of Virtual Reality (San Francisco, CA), 48-53.

Kilteni, K., Groten, R., and Slater, M. (2012). The Sense of embodiment in virtual reality. Presence Teleoper. Virtual Environ. 21, 373-387. doi: 10.1162/PRES_a_00124

Kokkinara, E., and Slater, M. (2014). Measuring the effects through time of the influence of visuomotor and visuotactile synchronous stimulation on a virtual body ownership illusion. Perception 43, 43-58. doi: 10.1068/p7545

Kopeć, W., Wichrowski, M., Kalinowski, K., Jaskulska, A., Skorupska, K., Cnotkowski, D., et al. (2019). VR with older adults: participatory design of a virtual atm training simulation. arXiv 52, 277-281. doi: 10.1016/j.ifacol.2019.12.110

LaViola, J. J. (2000). A discussion of cybersickness in virtual environments. ACM SIGCHI Bull. 32, 47-56. doi: 10.1145/333329.333344

Leeb, R., Friedman, D., Slater, M., and Pfurtscheller, G. (2012). "A tetraplegic patient controls a wheelchair in virtual reality. Brain-computer interfaces games work," in International Conference on Advances in Computer Entertainment Technology (Salzburg).

Leeb, R., and Pérez-Marcos, D. (2020). Brain-computer interfaces and virtual reality for neurorehabilitation. Handb. Clin. Neurol. 168, 183-197. doi: 10.1016/B978-0-444-63934-9.00014-7
Llobera, J., Sanchez-Vives, M. V., and Slater, M. (2013). The relationship between virtual body ownership and temperature sensitivity. J. R. Soc. Interface 10:20130300. doi: 10.1098/rsif.2013.0300

Lotte, F., Faller, J., Guger, C., Renard, Y., Pfurtscheller, G., Lécuyer, A., et al. (2012). "Combining BCI with virtual reality: towards new applications and improved BCI," in Towards Practical Brain-Computer Interfaces. Biological and Medical Physics, Biomedical Engineering, eds B. Allison, S. Dunne, R. Leeb, J. D. R. Millán, and A. Nijholt (Heidelberg: Springer), 197-220. doi: 10.1007/978-3-642-29746-5_10

Lotze, M., and Moseley, G. L. (2007). Role of distorted body image in pain. Curr. Rheumatol. Rep. 9, 488-496. doi: 10.1007/s11926-007-0079-x

Lupu, R. G., Irimia, D. C., Ungureanu, F., Poboroniuc, M. S., and Moldoveanu, A. (2018). BCI and FES based therapy for stroke rehabilitation using VR facilities. Wireless Commun. Mob. Comput. 2018:4798359. doi: 10.1155/2018/4798359

Maister, L., Slater, M., Sanchez-Vives, M. V., and Tsakiris, M. (2015). Changing bodies changes minds: owning another body affects social cognition. Trends Cogn. Sci. 19, 6-12. doi: 10.1016/j.tics.2014.11.001

Marasco, P. D. (2018). Using proprioception to get a better grasp on embodiment. J. Physiol. 596, 133-134. doi: 10.1113/JP275468

Matamala-Gomez, M., Donegan, T., Bottiroli, S., Sandrini, G., Sanchez-Vives, M. V., and Tassorelli, C. (2019). Immersive virtual reality and virtual embodiment for pain relief. Front. Hum. Neurosci. 13:279. doi: 10.3389/fnhum.2019. 00279

Matamala-Gomez, M., Gonzalez, A. M. D., Slater, M., Sanchez-Vives, M. V. V., Diaz Gonzalez, A. M., Slater, M., et al. (2018). Decreasing pain ratings in chronic arm pain through changing a virtual body: different strategies for different pain types. J. Pain 20, 685-697. doi: 10.1016/j.jpain.2018.12.001

Nierula, B., Martini, M., Matamala-Gomez, M., Slater, M., and Sanchez-Vives, M. V. (2017). Seeing an embodied virtual hand is analgesic contingent on colocation. J. Pain 18, 645-655. doi: 10.1016/j.jpain.2017.01.003

Nierula, B., and Sanchez-Vives, M. V. (2019). "Can BCI paradigms induce feelings of agency and responsibility over movements?" in Brain-Computer Interface Research, eds C. Guger, N. Mrachacz-Kersting, and B. Z. Allison (Cham: Springer), 103-114. doi: 10.1007/978-3-030-05668-1_10

Nierula, B., Spanlang, B., Martini, M., Borrell, M., Nikulin, V. V., and SanchezVives, M. V. (2019). Agency and responsibility over virtual movements controlled through different paradigms of brain-computer interface. J. Physiol. JP278167. doi: 10.1113/JP278167. [Epub ahead of print].

Ortner, R., Irimia, D., Scharinger, J., and Guger, C. (2012). A motor imagery based brain-computer interface for stroke rehabilitation. Stud. Health Technol. Inform. 181, 319-323. doi: 10.3233/978-1-61499-121-2-319

Perez-Marcos, D., Slater, M., and Sanchez-Vives, M. V. (2009). Inducing a virtual hand ownership illusion through a brain-computer interface. NeuroReport 20, 589-594. doi: 10.1097/WNR.0b013e32832a0a2a

Perez-Marcos, D., Solazzi, M., Steptoe, W., Oyekoya, O., Frisoli, A., Weyrich, T., et al. (2012). A fully immersive set-up for remote interaction and neurorehabilitation based on virtual body ownership. Front. Neurol. 3:110. doi: 10.3389/fneur.2012.00110

Remsik, A. B., Dodd, K., Leroy, W., Thoma, J., Jacobson, T., Allen, J. D., et al. (2018). Behavioral outcomes following brain-computer interface intervention for upper extremity rehabilitation in stroke: a randomized controlled trial. Front. Neurosci. 12:752. doi: 10.3389/fnins.2018.00752

Riva, G., Wiederhold, B. K., and Mantovani, F. (2018). Neuroscience of virtual reality: from virtual exposure to embodied medicine. Cyberpsychol. Behav. Soc. Netw. 22, 82-96. doi: 10.1089/cyber.2017.29099.gri

Sanchez-Vives, M. V., Spanlang, B., Frisoli, A., Bergamasco, M., and Slater, M. (2010). Virtual hand illusion induced by visuomotor correlations. PLoS ONE 5:e10381. doi: 10.1371/journal.pone.0010381

Schmitt, Y. S., Hoffman, H. G., Blough, D. K., Patterson, D. R., Jensen, M. P., Soltani, M., et al. (2011). A randomized, controlled trial of immersive virtual reality analgesia, during physical therapy for pediatric burns. Burns 37, 61-68. doi: 10.1016/j.burns.2010.07.007

Serrano, B., Baños, R. M., and Botella, C. (2016). Virtual reality and stimulation of touch and smell for inducing relaxation: a randomized controlled trial. Comput. Human Behav. 55, 1-8. doi: 10.1016/j.chb.2015.08.007

Sherman, W. R., and Craig, A. B. (2018). Understanding Virtual Reality: Interface, Application, and Design. San Francisco, CA: Morgan Kaufman. doi: 10.1016/B978-0-12-800965-9.00010-6 
Slater, M. (2009). Place illusion and plausibility can lead to realistic behaviour in immersive virtual environments. Philos. Trans. R. Soc. 364, 3549-3557. doi: $10.1098 /$ rstb. 2009.0138

Slater, M. (2014). Grand challenges in virtual environments. Front. Robot. AI 1:3. doi: 10.3389/frobt.2014.00003

Slater, M., Perez-Marcos, D., Ehrsson, H. H., and Sanchez-Vives, M. V. (2008). Towards a digital body: the virtual arm illusion. Front. Hum. Neurosci. 2:6. doi: 10.3389/neuro.09.006.2008

Slater, M., Perez-Marcos, D., Ehrsson, H. H., and Sanchez-Vives, M. V. (2009). Inducing illusory ownership of a virtual body. Front. Neurosci. 3:214-220. doi: 10.3389/neuro.01.029.2009

Slater, M., and Sanchez-Vives, M. V. (2016). Enhancing our lives with immersive virtual reality. Front. Robot. AI 3:74. doi: 10.3389/frobt.2016.00074

Solcà, M., Ronchi, R., Bello-Ruiz, J., Schmidlin, T., Herbelin, B., Luthi, F., et al. (2018). Heartbeat-enhanced immersive virtual reality to treat complex regional pain syndrome. Neurology 91, e1-e11. doi: 10.1212/WNL.0000000000005905

Soltani, M., Drever, S. A., Hoffman, H. G., Sharar, S. R., Wiechman, S. A., Jensen, M. P., et al. (2018). Virtual reality analgesia for burn joint flexibility: a randomized controlled trial. Rehabil. Psychol. 63, 487-494. doi: 10.1037/rep0000239

Spiss, S., Siess, M., Kim, Y., and Harders, M. (2018). "Effect of touch stimuli on proprioceptive recalibration during upper-limb rotation in virtual reality mirror therapy," in Proceedings of the IEEE RAS and EMBS International
Conference on Biomedical Robotics and Biomechatronics (IEEE Computer Society) (Enschede), 279-284. doi: 10.1109/BIOROB.2018.8488133

Stanton, T. R., Gilpin, H. R., Edwards, L., Moseley, G. L., and Newport, R. (2018). Illusory resizing of the painful knee is analgesic in symptomatic knee osteoarthritis. PeerJ. 6:e5206. doi: 10.7717/peerj.5206

Vourvopoulos, A., Jorge, C., Abreu, R., Figueiredo, P., Fernandes, J. C., and Bermúdez i Badia, S. (2019). Efficacy and brain imaging correlates of an immersive motor imagery BCI-driven VR system for upper limb motor rehabilitation: a clinical case report. Front. Hum. Neurosci. 13:244. doi: 10.3389/fnhum.2019.00244

Conflict of Interest: The authors declare that the research was conducted in the absence of any commercial or financial relationships that could be construed as a potential conflict of interest.

Copyright (c) 2020 Donegan, Ryan, Swidrak and Sanchez-Vives. This is an openaccess article distributed under the terms of the Creative Commons Attribution License (CC BY). The use, distribution or reproduction in other forums is permitted, provided the original author(s) and the copyright owner(s) are credited and that the original publication in this journal is cited, in accordance with accepted academic practice. No use, distribution or reproduction is permitted which does not comply with these terms. 